\title{
Diagnostic Dilemmas: A Multi-Institutional Retrospective Analysis of Adrenal Incidentaloma Pathology Based on Radiographic Size
}

\section{David Zekan ( $\sim$ dszekan@hsc.wvu.edu )}

West Virginia University Department of Urology, 1 Medical Center Drive, Morgantown, WV 26505

\section{Robert Scott King}

Charleston Area Medical Center Department of Urology, 3100 MacCorkle Ave SE Suite 602, Charleston, WV 25304

\section{Ali Hajiran}

West Virginia University Department of Urology, 1 Medical Center Drive, Morgantown, WV 26505

Apexa Patel

Charleston Area Medical Center Health Education and Research Institute, 3110 MacCorkle Ave SE, Charleston, WV 25304

\section{Samuel Deem}

Charleston Area Medical Center Department of Urology, 3100 MacCorkle Ave SE Suite 602, Charleston, WV 25304

\section{Adam Luchey}

West Virginia University Department of Urology, 1 Medical Center Drive, Morgantown, WV 26505

\section{Research Article}

Keywords: cross-sectional imaging, adrenocortical carcinoma, malignancy, CT, MRI

Posted Date: December 23rd, 2021

DOI: https://doi.org/10.21203/rs.3.rs-1150325/v1

License: (c) (i) This work is licensed under a Creative Commons Attribution 4.0 International License. Read Full License 


\section{Abstract}

\section{Introduction/Background}

Adrenal incidentalomas (Als) are masses $>1 \mathrm{~cm}$ found incidentally during radiographic imaging. They are present in up to $4.4 \%$ of patients undergoing CT scan, and incidence is increasing with usage and sensitivity of cross-sectional imaging. Most result in diagnosis of adrenal cortical adenoma, questioning guidelines recommending removal of all Als with negative functional workup. This retrospective study analyzes histological outcome based on size of non-functional adrenal masses.

\section{Material and Methods}

10 years of data was analyzed from two academic institutions. Exclusion criteria included patients with positive functional workups, those who underwent adrenalectomy during nephrectomy, <18 years, and incomplete records. Al radiologic and histologic size, histologic outcome, laterality, imaging modality, gender, and age were collected. T-test was used for comparison of continuous variables, and the twosided Fisher's exact or chi-square test were used to determine differences for categorical variables. Univariate analysis of each independent variable was performed using simple logistic regression.

\section{Results}

73 adrenalectomies met the above inclusion criteria. 60 were detected on CT scan, 12 on MRI, and one on ultrasound. Eight of 73 cases resulted in malignant pathology, 3 of which were adrenocortical carcinoma (ACC). Each ACC measured $>6 \mathrm{~cm}$, with mean radiologic and pathologic sizes of $11.2 \mathrm{~cm}$ and $11.3 \mathrm{~cm}$. Both radiologic and pathologic size were significant predictors of malignancy ( $p=0.008$ and 0.011$)$.

\section{Conclusions}

Our results question the generally-accepted $4 \mathrm{~cm}$ cutoff for excision of metabolically-silent Als. They suggest a $6 \mathrm{~cm}$ threshold would suffice to avoid removal of benign lesions while maintaining sensitivity for ACC.

\section{Introduction}

The malignant potential of a small, incidentally found lesion of the adrenal gland causes great anxiety amongst patients, but rarely causes harm. An adrenal incidentaloma (Al) is defined as mass measuring greater than $1 \mathrm{~cm}$ on the adrenal gland found fortuitously during radiographic imaging. ${ }^{1}$ The diameter of the average Al measures approximately 3 to $3.5 \mathrm{~cm}$ on Computed Tomography (CT) scan. ${ }^{2}$ With 
increased utilization and advancements in the sensitivity of medical imaging, adrenal masses are observed in as many as $4.4 \%$ of all patients undergoing CT scan. ${ }^{3}$

Over the past two decades, an increasing body of evidence has supported restructuring the current guidelines for the management of Als. Most Als result in the histological diagnosis of benign adrenal cortical adenoma. ${ }^{2}$ Adrenocortical carcinoma (ACC) is more commonly diagnosed at younger ages than benign adrenal masses and is found more often in males than females. ${ }^{2}$ For patients without a history of malignancy, the estimated prevalence of ACC is less than 2 cases per million adrenal lesions identified. ${ }^{4}$ Twenty-five percent of benign masses increase in size during follow up; however, given the estimated 1 in 1000 risk of malignant transformation over time for benign masses, continued observation may be an appropriate option. ${ }^{2}$ Exposing patients to additional risks with surgery for a rare chance of cancer in smaller adrenal masses may not be warranted.

The American Association of Clinical Endocrinologists (AACE) and American Association of Endocrine Surgeons (AAES) guidelines have changed over the last decade in regards to excision based on size from a previous cutoff of $\geq 6 \mathrm{~cm}$ to now only $>4 \mathrm{~cm} .{ }^{5}$ Current clinical guidelines recommend a workup to include contrasted cross-sectional imaging and a functional evaluation to investigate for hormonal activity. Surgical excision is then recommended if the lesion is radiographically suspicious, $>4 \mathrm{~cm}$ in size, or hormonally active. ${ }^{6}$ Due to the rarity of ACC, most of these recommendations are based on information obtained from controlled trials without randomization (Level 3 Evidence) ${ }^{5}$

Because there have been few randomized controlled studies regarding the management of Als, there is a lack of qualified management guidelines for patient care. ${ }^{6}$ To address this clinical question, we sought to perform a collaborative effort reviewing ten years of adrenalectomy data comparing pathologic outcomes based on size criteria. It was hypothesized that our data would further support a needed modification in the current guidelines for removal of Als.

\section{Materials And Methods}

Following approval from respective institutional review boards, including waivers of informed consent from West Virginia University Institutional Review Board and the CAMC/WVU-Charleston Division Institutional Review Board for the Protection of Human Subjects, a two-institution retrospective chart review of adrenalectomies over 10 years was performed. Patients who underwent adrenalectomy at two tertiary care centers, Charleston Area Medical Center (CAMC) and West Virginia University (WVU), from January 1, 2005 through December 31, 2015 were included. Patients who underwent adrenalectomy during nephrectomy, were less than 18 years of age, or had incomplete medical records were excluded. Data analysis was then performed on those with available cross-sectional imaging who underwent adrenalectomy with no history of malignancy and a negative functional workup.

The Center for Health Service and Outcomes Research at CAMC conducted data analysis. For this exploratory study, selection of appropriate statistical measures was determined by CAMC's Center for 
Health Education and Research Institute. Two-tailed chi-square alpha was set at 0.05. T-test was used for comparison of continuous variables, and the two-sided Fisher's exact or chi square test were used to determine differences for categorical variables. Univariate analysis of each independent variable was performed using simple logistic regression. A variable with a univariate $p<0.10$ was included in the model for multivariate analysis. A $p$-value $<0.05$ was considered statistically significant.

\section{Results}

A total of 234 patients underwent adrenalectomy from January 1, 2005 to December 31, 2015. 161 cases were excluded: 61 hormonally active lesions (based on pre-operative testing), 55 known metastases, 10 adrenalectomies during resection of a primary renal lesion, and 35 with inadequate records or $<18$ years of age.

There were 73 adrenalectomies performed solely based on size criteria of the adrenal mass $(1.7-22.8 \mathrm{~cm})$, as opposed to metabolically active lesions. The average age was 56.8 years with M:F ratio of $0.62: 1$. One patient in the analyzed group had bilateral masses, while 44 had left-sided masses and 28 had rightsided masses. 60 of the masses were determined appropriate for resection based on CT scan, while 12 were detected on MRI, and one was seen only on ultrasound. In all but 8 of the 73 total adrenalectomies performed based on size criteria alone, final pathology reported benign histology. Three of these cases would result in ACC, each measuring $\geq 6 \mathrm{~cm}$; all (53 total) masses measuring $<6 \mathrm{~cm}$ removed solely on the basis of size criteria were benign aside from a single metastasis (figure 1).

The ACC mean radiographic and pathologic size was $11.2 \mathrm{~cm}$ and $12.3 \mathrm{~cm}$, respectively. ACCs accounted for $14.2 \%$ of the 21 adrenalectomies performed for masses $\geq 6 \mathrm{~cm}$.

Thus, $\geq 6 \mathrm{~cm}$ was deemed an appropriate cutoff for data analysis for the purposes of this study. Neither gender nor age were significant predictors of the adrenal mass size category $(<6 \mathrm{~cm}$ or $\geq 6 \mathrm{~cm}$; determined both radiologically and pathologically as the two were not always concordant of patients in this study (Table 1).

Table 1

Radiologic and Pathologic Size Discordance Amongst Adrenal Incidentalomas: demonstration of statistically significant discordance between the radiologic (pre-operative) and pathologic (post-operative) sizes of adrenal incidentalomas included in our analysis.

\begin{tabular}{|llll|}
\hline Pathologic Size $(\mathrm{cm})$ & \multicolumn{2}{l|}{ Radiographic Size $(\mathrm{cm})$} & p-value \\
\cline { 2 - 3 } & $<6$ & $\geq 6$ & \\
\hline$<6$ & $46(88.46)$ & $1(4.76)$ & $<0.0001$ \\
\hline$\geq 6$ & $6(11.54)$ & $20(95.24)$ & \\
\hline
\end{tabular}

However, size was predictive of pathology, most importantly malignancy ( $p=0.008$ and 0.011 , Table 2). 
Table 2

Demographics and Pathology Based on Radiologic and Pathologic Size

\begin{tabular}{|c|c|c|c|c|}
\hline & & $<6$ & $\geq 6$ & $\begin{array}{l}\text { p- } \\
\text { value }\end{array}$ \\
\hline \multirow[t]{3}{*}{ Radiological Size (cm) } & Male & $20(38.46)$ & $8(38.10)$ & \multirow[t]{2}{*}{0.976} \\
\hline & Female & $32(61.54)$ & $13(61.90)$ & \\
\hline & Age (years) & $59.09 \pm 11.81$ & $56.33 \pm 11.98$ & 0.390 \\
\hline \multirow[t]{4}{*}{$\begin{array}{l}\text { Pathologic Status Based on } \\
\text { Radiologic Size }\end{array}$} & $\begin{array}{l}\text { Adrenocortical } \\
\text { carcinoma }\end{array}$ & 0 & $3(14.29)$ & \multirow[t]{4}{*}{$0.008^{*}$} \\
\hline & Kidney cancer & 0 & $1(4.76)$ & \\
\hline & Benign adenoma & $50(96.15)$ & $15(71.43)$ & \\
\hline & $\begin{array}{l}\text { Metastatic from other } \\
\text { organ }\end{array}$ & $2(3.85)$ & $2(9.52)$ & \\
\hline \multirow[t]{3}{*}{ Pathological Size (cm) } & Male & $18(38.30)$ & $10(38.46)$ & \multirow[t]{2}{*}{0.989} \\
\hline & Female & $29(61.70)$ & $16(61.54)$ & \\
\hline & Age (years) & $58.95 \pm 12.01$ & $56.96 \pm 11.66$ & 0.494 \\
\hline \multirow[t]{4}{*}{$\begin{array}{l}\text { Pathologic Status Based on } \\
\text { Pathologic Size }\end{array}$} & $\begin{array}{l}\text { Adrenocortical } \\
\text { carcinoma }\end{array}$ & 0 & $3(11.54)$ & \multirow[t]{4}{*}{$0.011^{*}$} \\
\hline & Kidney cancer & 0 & $1(3.85)$ & \\
\hline & Benign adenoma & $46(97.87)$ & 19 (73.08) & \\
\hline & $\begin{array}{l}\text { Metastatic from other } \\
\text { organ }\end{array}$ & $1(2.13)$ & $3(11.54)$ & \\
\hline
\end{tabular}

Namely, all adrenocortical carcinomas (3), apparent adrenal masses on pre-operative imaging that were found to be renal cell carcinomas pathologically (1), and $50 \%$ of adrenal masses that were incidentally found to be metastases ( 2 of 4 ) fell into the $\geq 6 \mathrm{~cm}$ category. Of the 55 masses resected based on size alone that showed adrenal adenoma as the final pathology, only 19 fell into the $\geq 6 \mathrm{~cm}$ category (34.5\%) (figure 1).

\section{Discussion}

Continued innovation in imaging modalities has enhanced sensitivity in identifying abnormalities of the adrenal glands. The prevalence of adrenal adenomas in the general population is estimated at $3-7 \%$, with the overwhelming majority of those discovered incidentally demonstrating benign pathology. ${ }^{7}$ However, because the risk of primary adrenocortical carcinoma is reported to be as high as $4.7 \%$ in patients with incidentally discovered adrenal masses, characterization and appropriate treatment remain crucial. ${ }^{8}$ The prevalence of clinically unapparent adrenal masses at autopsy is reported at nearly $2.1 \%$. Detection is 
increasing with the advent of modern imaging technology. Estimates of incidence range from $0.1 \%$ for general health ultrasound screening to $0.42 \%$ in patients evaluated for non-endocrine symptoms to $4.3 \%$ in patients previously diagnosed with cancer. This prevalence also varies with age, at $<1 \%$ for patients younger than 30 years of age and increasing to $7 \%$ in patients 70 years of age or older. ${ }^{9}$ Overall, incidentally identified adrenal masses occur in up to $9 \%$ of CT scans, justifying the need for accurate guidelines to direct their management. ${ }^{10}$

Advancements in imaging also allow for better characterization of adrenal masses prior to biopsy or surgical resection. Technological developments have improved the anatomic resolution, sensitivity, and specificity of cross-sectional imaging modalities, particularly MRI and contrasted CT (recommended modalities in the setting of $\mathrm{Al}$ ), over the last $15-20$ years. ${ }^{5}$ This is fueled by the superiority of these modalities over their conventional counterparts in the accurate detection of disease. Similarly, advancements have led to an increase in data generated with more cross-sectional images per scan. ${ }^{11}$ Improved cross-sectional imaging allows for detection of intralesional fat, water and blood enabling characterization of benign adrenal masses, such as adenomas, angiomyolipomas, cysts, granulomas and hemorrhage. Nieman et al. emphasize the importance of washout in delayed-phase CT scan due to the high prevalence of benign histology in masses with washout $>50 \% .{ }^{12,13}$ When inconclusive, or if concerning features are noted, it may be appropriate to establish a tissue diagnosis by performing adrenal biopsy or perform definitive surgical management for potentially malignant lesions. Accurate characterization is imperative due to the potential for metastases to the adrenal gland, which may preclude surgical or radiation therapy to another primary site. ${ }^{10}$

An additional factor contributing to increased detection is increased utilization of topographic imaging. In 2008, an estimated 60 million CT scans were performed in the United States growing at a rate of $5 \%$ per year. ${ }^{14}$ This is astounding when compared to the $3.8 \%$ rate of increased imaging amongst Medicare patients between 1993-1999 and parallels the increase in technology occurring with cross-sectional imaging. ${ }^{15}$ This widespread use of cross-sectional imaging is thought to have occurred because of ordering physicians' attempts to address demands of both consumers and referring physicians, while providing superior patient care. ${ }^{14} \mathrm{~A}$ significant proportion derives from a somewhat overzealous usage of cross-sectional imaging in emergency departments. ${ }^{16}$ A 2013 study revealed that CT technology is available in $97 \%$ of emergency department (ED) visits in the United States, and is performed during $11.4 \%$ of visits, most commonly for complex abdominal pain. ${ }^{17}$ Similarly, Bellalio et al. report an almost $60 \%$ increase in CT utilization in ED's from 2005-2013 with an order for CT in 17.8\% of visits. The only population that saw a decrease in utilization was children, who were not included in our study and are less likely to have adrenal incidentalomas. ${ }^{16}$ These data are corroborated by Baloescu, who underscores that even with greater than 60 million CT scans ordered in ED's in the United States in 2005, no decrease in morbidity and mortality was appreciated. ${ }^{18}$ It is less likely that this increase in cross-sectional imaging is coming from primary care providers in the outpatient setting, as Weilburg et al. demonstrate the ability of a utilization management system to decrease the number of high cost imaging studies (CT, MRI, 
nuclear imaging, and PET) ordered in the outpatient setting from 0.43 exams per year in 2007 to 0.34 exams per year in 2013 , with a cohort of around 100,000 patients. ${ }^{19}$ Clearly, it is possible to implement systems in which the over-utilization of cross-sectional imaging is curtailed, but it remains prevalent in EDs and other clinical settings, including inpatient admissions, leading to the common discovery of Als highlighted in our study.

With increased detection comes an increased demand for evidence-based clinical recommendations to appropriately guide health care personnel in the management of Als. Multiple authors have demonstrated the benign nature of the majority of small adrenal masses. The AACE and AAES published guidelines a decade ago highlighting their recommendations for workup and management of such lesions. In short, they recommend clinical, biochemical, and radiologic evaluation of patients with signs of hypercortisolism, hyperaldosteronism (if hypertensive), pheochromocytoma, or malignancy. Adrenalectomy is recommended after hormonal evaluation in patients with a mass $\geq 4 \mathrm{~cm}$ or with malignant features on CT scan. Adrenalectomy is also recommended in smaller, less suspicious tumors in the presence of hormonal abnormalities as evidenced by aldosterone concentration/renin activity, plasma-free metanephrines and normetanephrines, and overnight 1-mg dexamethasone suppression test. Patients in whom adrenalectomy is not recommended $(<4 \mathrm{~cm}$ homogenous lesions with regular borders and $<10 \mathrm{HU}$ on non-contrast CT) should follow surveillance guidelines, which are discussed below. ${ }^{5}$

The $4 \mathrm{~cm}$ cutoff for adrenalectomy is well-established and perhaps too-readily accepted by those outlining guidelines for the management of Als. Mantero et al. reported that $4 \mathrm{~cm}$ had a greater sensitivity (93\%) for detection of ACC when compared to $5 \mathrm{~cm}(81 \%)$ and $6 \mathrm{~cm}(74 \%)$, respectively. However, their 2000 study was limited to an Italian population that included only 1004 patients and the issue of size's ability to predict benign or malignant pathology has been revisited little in the past two decades. ${ }^{20}$ Sturgeon et al. evaluated 457 patients with ACC and 47 patients with adrenal adenomas, establishing the sensitivity of tumor size in predicting malignancy to be $96 \%$ for tumors $\geq 4 \mathrm{~cm}, 90 \%$ for tumors $\geq 6 \mathrm{~cm}$, and $77 \%$ for tumors $\geq 8 \mathrm{~cm}$. Based on their data, at a threshold of $\geq 4 \mathrm{~cm}$, the likelihood of malignancy doubles (to $10 \%) .{ }^{21}$ Cutoffs ranging from 4-6 cm have been proposed for surgical excisional of incidentally-discovered adrenal masses. A recent analysis of 2219 patients by Kahramangil et al. shows ACC rates of $0.1 \%, 2.4 \%$, and $19.5 \%$ in patients with masses $<4 \mathrm{~cm}, 4-6 \mathrm{~cm}$, and $>6 \mathrm{~cm}$, respectively, with an optimal cutoff of $4.6 \mathrm{~cm}$. In addition, analysis by Hounsfield (HU) density on non-contrast CT showed ACC risk of $0 \%, 0.5 \%$, and $6.3 \%$ for masses of $<10,10-20$, and $>20 \mathrm{HU}$, respectively. In addition, male sex and $>0.6 \mathrm{~cm} /$ year growth rate were independent predictors of ACC. ${ }^{22}$ Similarly, Birsen et al. (2014) describe development of a scoring system to determine the probability of an adrenal mass representing ACC based on size and HU. Of their 157 patients, seven without hormonal secretion had ACC on final pathology; of these, only a single ACC measured $<6 \mathrm{~cm} .{ }^{23}$ Due to increasing risk of malignancy with those $>4 \mathrm{~cm}$, authors readily accept $4 \mathrm{~cm}$ as the cutoff. However, our data suggests that this cutoff may be overly-cautious and subjects patients to unnecessary morbidity that accompanies adrenalectomy. 
Along with a conservative approach to management comes a need to clarify ongoing surveillance imaging and laboratory evaluation intervals. This is due to a $17 \%, 29 \%, 47 \%$ risk of Als converting to functional status and a $6 \%, 14 \%, 29 \%$ risk of increasing in size at 1,2 , and 5 years, respectively. ${ }^{5}$ Current guidelines vary, with most suggesting follow-up guided by clinical judgment and the presumed cause of the mass based on initial workup. National Institute of Health $(\mathrm{NIH})$ data suggests that for masses that are benign-appearing $(<10 \mathrm{HU}$; washout $>50 \%)$, small $(<3 \mathrm{~cm})$, and completely non-functioning, imaging and biochemical reevaluation at 1-2 years (or more) is appropriate, with subsequent follow-up only if the clinical picture changes; the risk of malignancy or subsequent hyperfunction is almost nonexistent though reported conversion to ACC exists in recent literature. ${ }^{24}$ For indeterminate lesions, repeat evaluation for growth after 3-12 months is appropriate. Subsequent testing should occur earlier for lesions with increasing size, and later for those with no change. ${ }^{12}$ The AAES extends the criteria for "small" Als to include those $<4 \mathrm{~cm}$ and they better outline surveillance recommendations to include radiographic reevaluation at 3 to 6 months, then annually for 1 to 2 years. Similarly, they suggest hormonal evaluation annually for up to 5 years. ${ }^{5}$ Repeat screening for hyperaldosteronism is not perceived to be beneficial, but most authors recommend screening for catecholamine and cortisol excess for at least four years due to the similar appearance of pheochromocytomas to lipid-poor adenomas on CT scan. This is one instance in which clinical judgment can be used to guide surveillance as most pheochromocytomas grow over time suggesting that further imaging may not be indicated in the setting of a stable mass. ${ }^{12}$

The above algorithms include 1-3 radiological assessments in the first two years with reconsideration of surgical excision with 0.5-1 cm of growth during follow up. Corwin et al. (2019) assessed 131 adrenal masses, 26 of which were found to be malignant and 121 adenoma. Of these, all malignant nodules increased in size during follow-up, with a mean growth of $5.8 \mathrm{~cm} /$ year. A growth rate of $3 \mathrm{~mm} /$ year distinguished adenomas from malignant nodules with sensitivity and specificity of $100 \%{ }^{25}$ Repeating the same imaging modality (generally CT scan) is encouraged to assess for changes in the mass. ${ }^{6}$ The American College of Radiology better defines imaging protocols, including measurement of density and contrast washout, as well as the importance of clinical correlation when determining surveillance regimens for Als $<4 \mathrm{~cm}$. Specifically, in 2-4 cm Als, an adrenal CT protocol should be used at diagnosis to better characterize the lesion, while lesions $<2 \mathrm{~cm}$ may have an adrenal CT protocol at one year to document stability. Chomsky-Higgins et al. performed a cost-effectiveness analysis of surveillance of adrenal masses 1-4 cm with an initial normal workup including non-contrasted CT showing a mass $<10$ $\mathrm{HU}$, no suspicious features, and negative hormonal evaluation. They determined it is most effective to perform a single follow up at one year with non-contrasted CT and biochemical evaluation. ${ }^{26}$ It may be reasonable to consider excision in those adrenal masses with indeterminate radiologic features that grow at least $0.8 \mathrm{~cm}$ during 3-12 month follow-up. ${ }^{13}$ Post-excision surveillance for Als $>4 \mathrm{~cm}$ remains poorly defined and is based on the histological diagnosis at the time of extirpation. ${ }^{27}$ Although recommendations for radiologic follow-up of nonfunctional adrenal masses vary based on the organization, it is important to consider that frequent adrenal imaging is associated with additional cost, 
anxiety, and exposure to radiation, which may theoretically induce cancer at an estimated rate similar to the chance of developing adrenal malignancy. ${ }^{13}$

Limitations of this study include its retrospective nature, which has the potential to introduce biases in data collection and analysis. Although it is a multi-institutional study, both institutions are within the same geographic region with a notably homogenous population, limiting its applicability to wider groups. Also, the lack of surveillance data within the population limits our ability to detect recurrence amongst participants and growth of non-operative Als. Similarly, surgical patients are the only subjects included, limiting the ability to assess cancer risk in a more specific population of patients on active surveillance who undergo resection following Al growth. However, the above study design achieves the goal of assessing pathological outcomes in patients undergoing adrenalectomy for incidentally detected adrenal masses. Addition of patients to our study data from institutions with more diverse populations in terms of race and age as well as surveillance data provide future directions for this research.

\section{Conclusions}

Regarding surgical excision of an $\mathrm{Al}$, the decision to operate is difficult in a patient with no known cancer history, a negative hormonal evaluation, and inconclusive radiographic characteristics. The findings of this study suggest that a $6 \mathrm{~cm}$ threshold for surgical excision may be considered to avoid removal of benign lesions while maintaining an acceptable sensitivity for adrenocortical carcinoma. Significant overlap exists between guidelines for surveillance of adrenal masses. Further investigation is warranted to establish evidence-based recommendations which more thoroughly outline surveillance protocols of Als.

\section{Declarations}

\section{Ethics Approval and Consent}

This study was approved by the West Virginia University Institutional Review Board and the CAMC/WVUCharleston Division Institutional Review Board for the Protection of Human Subjects, respectively, and was performed in accordance with relevant guidelines and regulations set forth by these governing bodies.

\section{Consent for Publication}

Not applicable

\section{Availability of Data and Materials}

The datasets used and/or analyzed during the current study are available from the corresponding author on reasonable request. 
The authors declare that they have no competing interests.

\section{Funding}

This research and manuscript received no funding.

\section{Author's contributions}

David Zekan, MD- data collection, analysis and manuscript preparation/editing

Robert Scott King, DO- IRB, data collection, analysis and manuscript preparation/editing

Ali Hajiran, MD- IRB, data collection, analysis and manuscript preparation/editing

Apexa Patel, MD - data analysis and manuscript preparation/editing

Samuel Deem, DO- IRB, data collection, analysis and manuscript preparation/editing

Adam Luchey, MD `IRB, data collection, analysis and manuscript preparation/editing

\section{Acknowledgements}

Not applicable.

\section{References}

1. Young Jr WF. The incidentally discovered adrenal mass. New England Journal of Medicine. 2007 Feb 8;356(6):601-10.

2. Barzon L, Sonino N, Fallo F, Palu G, Boscaro M. Prevalence and natural history of adrenal incidentalomas. European journal of endocrinology. 2003 Oct 1;149(4):273-86.

3. Bovio S, Cataldi A, Reimondo G, Sperone P, Novello S, Berruti A, Borasio P, Fava C, Dogliotti L, Scagliotti GV, Angeli A. Prevalence of adrenal incidentaloma in a contemporary computerized tomography series. Journal of endocrinological investigation. 2006 Apr;29(4):298-302.

4. Mansmann G, Lau J, Balk E, Rothberg M, Miyachi Y, Bornstein SR. The clinically inapparent adrenal mass: update in diagnosis and management. Endocrine reviews. 2004 Apr 1;25(2):309-40.

5. Zeiger MA, Thompson GB, Duh QY, Hamrahian AH, Angelos P, Elaraj D, Fishman E, Kharlip J, Garber JR, Mechanick JI, Demeure MJ. American Association of Clinical Endocrinologists and American Association of Endocrine Surgeons medical guidelines for the management of adrenal incidentalomas. Endocrine practice. 2009 Jul 1;15:1-20.

6. Kapoor A, Morris T, Rebello R. Guidelines for the management of the incidentally discovered adrenal mass. Canadian Urological Association Journal. 2011 Aug;5(4):241.

7. Francis IR, Mayo-Smith WW. Adrenal Imaging. 2018 Mar 21. In: Hodler J, Kubik-Huch RA, von Schulthess GK. Diseases of the Abdomen and Pelvis 2018-2021: Diagnostic Imaging-IDKD Book. 
8. Choyke PL. ACR Appropriateness Criteria ${ }^{\circledR}$ on incidentally discovered adrenal mass. Journal of the American College of Radiology. 2006 Jul 1;3(7):498-504.

9. Grumbach MM, Biller BM, Braunstein GD, Campbell KK, Carney JA, Godley PA, Harris EL, Lee JK, Oertel YC, Posner MC, Schlechte JA. Management of the clinically inapparent adrenal mass (incidentaloma). Annals of internal medicine. 2003 Mar 4;138(5):424-9.

10. Sahdev A, Reznek RH. Imaging evaluation of the non-functioning indeterminate adrenal mass. Trends in Endocrinology \& Metabolism. 2004 Aug 1;15(6):271-6.

11. McDonald RJ, Schwartz KM, Eckel LJ, Diehn FE, Hunt CH, Bartholmai BJ, Erickson BJ, Kallmes DF. The effects of changes in utilization and technological advancements of cross-sectional imaging on radiologist workload. Academic radiology. 2015 Sep 1;22(9):1191-8.

12. Nieman LK. Approach to the patient with an adrenal incidentaloma. The Journal of Clinical Endocrinology \& Metabolism. 2010 Sep 1;95(9):4106-13.

13. Zeiger MA, Siegelman SS, Hamrahian AH. Medical and surgical evaluation and treatment of adrenal incidentalomas. The Journal of Clinical Endocrinology \& Metabolism. 2011 Jul 1;96(7):2004-15.

14. Boland GW, Guimaraes AS, Mueller PR. The radiologist's conundrum: benefits and costs of increasing CT capacity and utilization. European radiology. 2009 Jan;19(1):9-11.

15. Maitino AJ, Levin DC, Parker L, Rao VM, Sunshine JH. Nationwide trends in rates of utilization of noninvasive diagnostic imaging among the Medicare population between 1993 and 1999. Radiology. 2003 Apr;227(1):113-7.

16. Bellolio MF, Heien HC, Sangaralingham LR, Jeffery MM, Campbell RL, Cabrera D, Shah ND, Hess EP. Increased computed tomography utilization in the emergency department and its association with hospital admission. Western Journal of Emergency Medicine. 2017 Aug;18(5):835.

17. Berdahl CT, Vermeulen MJ, Larson DB, Schull MJ. Emergency department computed tomography utilization in the United States and Canada. Annals of emergency medicine. 2013 Nov 1;62(5):48694.

18. Baloescu C. Diagnostic imaging in emergency medicine: how much is too much?. Annals of emergency medicine. 2018 Dec 1;72(6):637-43.

19. Weilburg JB, Sistrom CL, Rosenthal DI, Stout MB, Dreyer KJ, Rockett HR, Baron JM, Ferris TG, Thrall $\mathrm{JH}$. Utilization management of high-cost imaging in an outpatient setting in a large stable patient and provider cohort over 7 years. Radiology. 2017 Sep;284(3):766-76.

20. Mantero F, Terzolo M, Arnaldi G, Osella G, Masini AM, Ali A, Giovagnetti M, Opocher G, Angeli A, Study Group on Adrenal Tumors of the Italian Society of Endocrinology. A survey on adrenal incidentaloma in Italy. The Journal of Clinical Endocrinology \& Metabolism. 2000 Feb 1;85(2):637-44.

21. Sturgeon C, Shen WT, Clark OH, Duh QY, Kebebew E. Risk assessment in 457 adrenal cortical carcinomas: how much does tumor size predict the likelihood of malignancy?. Journal of the American College of Surgeons. 2006 Mar 1;202(3):423-30.

22. Kahramangil B, Kose E, Remer EM, Reynolds JP, Stein R, Rini B, Siperstein A, Berber E. A modern assessment of cancer risk in adrenal incidentalomas: analysis of 2219 patients. Annals of surgery. 
2020 Jun 11.

23. Birsen O, Akyuz M, Dural C, Aksoy E, Aliyev S, Mitchell J, Siperstein A, Berber E. A new risk stratification algorithm for the management of patients with adrenal incidentalomas. Surgery. 2014 Oct 1;156(4):959-66.

24. Gagnon N, Boily P, Alguire C, Corbeil G, Bancos I, Latour M, Beauregard C, Caceres K, El Haffaf Z, Saad F, Olney HJ. Small adrenal incidentaloma becoming an aggressive adrenocortical carcinoma in a patient carrying a germline APC variant. Endocrine (1355008X). 2020 Apr 1;68(1).

25. Corwin MT, Navarro SM, Malik DG, Loehfelm TW, Fananapazir G, Wilson M, Campbell MJ.

Differences in growth rate on CT of adrenal adenomas and malignant adrenal nodules. American Journal of Roentgenology. 2019 Sep;213(3):632-6.

26. Chomsky-Higgins K, Seib C, Rochefort H, Gosnell J, Shen WT, Kahn JG, Duh QY, Suh I. Less is more: cost-effectiveness analysis of surveillance strategies for small, nonfunctional, radiographically benign adrenal incidentalomas. Surgery. 2018 Jan 1;163(1):197-204.

27. Mayo-Smith WW, Song JH, Boland GL, Francis IR, Israel GM, Mazzaglia PJ, Berland LL, Pandharipande PV. Management of incidental adrenal masses: a white paper of the ACR Incidental Findings Committee. Journal of the American College of Radiology. 2017 Aug 1;14(8):1038-44.

\section{Figures}

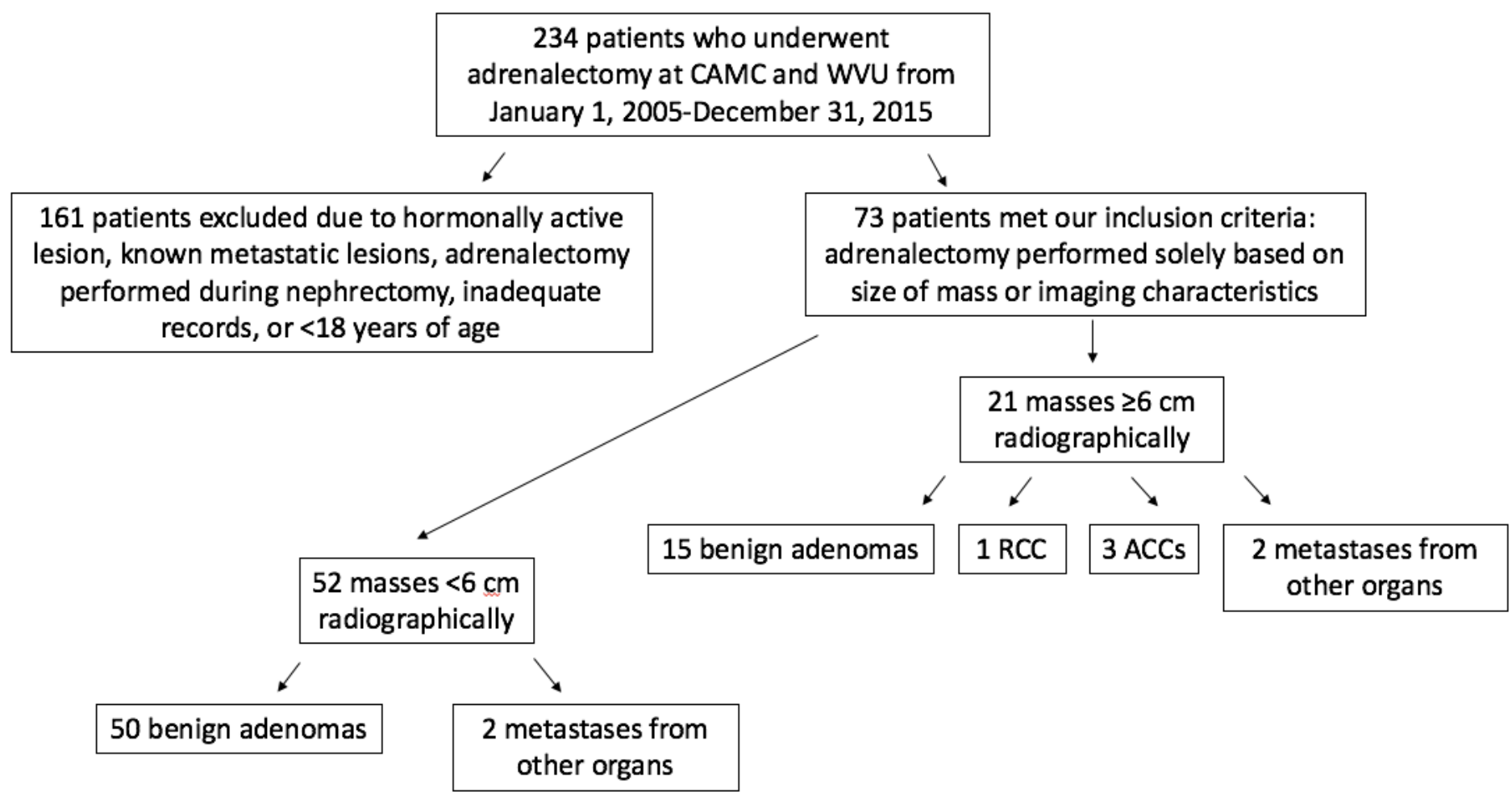

Figure 1 
Pathologic breakdown based on radiologic size of adrenal incidentalomas. RCC=renal cell carcinoma, ACC=adrenocortical carcinoma. 